\title{
Directed attention prolongs the perceived duration of a brief stimulus
}

\author{
STEFAN MATTES and ROLF ULRICH \\ University of Wuppertal, Wuppertal, Germany
}

\begin{abstract}
Stelmach, Herdman, and McNeil (1994) suggested recently that the perceived duration for attended stimuli is shorter than that for unattended ones. In contrast, the attenuation hypothesis (Thomas \& Weaver, 1975) suggests the reverse relation between directed attention and perceived duration. We conducted six experiments to test the validity of the two contradictory hypotheses. In all the experiments, attention was directed to one of two possible stimulus sources. Experiments 1 and 2 employed stimulus durations from 70 to $270 \mathrm{msec}$. A stimulus appeared in either the visual or the auditory modality. Stimuli in the attended modality were rated as longer than stimuli in the unattended modality. Ex periment 3 replicated this finding using a different psychophysical procedure. Experiments $4-6$ showed that the finding applies not only to stimuli from different sensory modalities but also to stimuli appearing at different locations within the visual field. The results of all six experiments support the assumption that directed attention prolongs the perceived duration of a stimulus.
\end{abstract}

Since the influential monograph by James (1890), there has been agreement that attention plays an important role in the perception of time (Brown \& West, 1990). Specifically, subjects estimate an interval to be shorter when an additional task requires the processing of nontemporal information. For example, in the classical studies of Katz (1906), intervals of about $1.2 \mathrm{sec}$ were judged shorter when attention was distracted by an accompanying task (e.g., reading of syllables). Meanwhile, numerous studies have replicated and extended this basic finding with various dual-task paradigms (Grondin \& Macar, 1992; Hülser, 1924; Macar, Grondin, \& Casini, 1994; Predebon, 1996; Quasebarth, 1924; Thomas \& Cantor, 1978; Underwood \& Swain, 1973; Zakay, 1993; Zakay \& Tsal, 1989).

For example, Macar et al. (1994) employed an attentionalsharing method. In each trial, several words appeared within an interval of 12 or $18 \mathrm{sec}$. The words came from different semantic categories and the subjects' task was to count animal names and to reproduce the duration of the word series at the end of the trial. Subjects were asked to divide their attention between the two tasks in prespecified proportions. When more attention was devoted to the counting task, subjects underestimated the duration of the word series. In a further experiment, the authors employed a discrimination task in which subjects were presented with stimuli of varying duration and intensity and judged both stimulus dimensions. As in the

Parts of this paper were presented at the annual meetings of the International Society for Psychophysics, 1994 (Vancouver, Canada) and 1996 (Padua, Italy). We thank Hiltraut Müller-Gethmann for her comments on an earlier version of this article and Lloyd L. Avant, Jim Enns, and Ray Klein for their insightful reviews. Correspondence should be addressed to S. Mattes, General Psychology I, Fachbereich 3, University of Wuppertal, Gauss-Strasse 20, D-42097 Wuppertal, Germany (email:mattes@uni-wuppertal.de). previous experiment, the subjects were asked to devote different proportions of attention to the two stimulus dimensions. Again, the duration was underestimated when more attention had to be allocated to the nontemporal task. These results demonstrate that the allocation of attention to a nontemporal task shortens the perceived duration of an event.

The authors interpreted this attentional effect in terms of the attentional model of time perception proposed by Thomas and Weaver (1975). This model assumes that the timing of an interval to be judged is performed by an internal timer that conceptually corresponds to counting models like those of Creelman (1962) or Treisman (1963). Counting models assume that an independent pacemaker generates pulses, which are accumulated during the stimulus interval to be judged. The more pulses the accumulation device counts during the interval, the longer is its perceived duration. According to Thomas and Weaver's attenuation hypothesis, the processing of nontemporal information detracts attention from the timer, and consequently the stream of pulses is attenuated. Thus, when limited attentional resources (Kahneman, 1973) have to be shared between temporal and nontemporal information processing, an interval is perceived as shorter the more attention is devoted to the nontemporal task.

In the studies just cited, attention had to be shared between a temporal and a nontemporal task. However, it is unclear whether a similar attentional effect results when attention has to be directed in advance to one of two possible stimulus sources instead of being shared between a temporal and a nontemporal task. For example, a brief visual stimulus may appear at an expected or an unexpected location within the visual field, and in each case the subject is asked to judge the duration of the stimulus. If the need to switch attention to the less probable stimulus source takes extra resources, less attention may be 
available for the timer and, consequently, fewer pulses are registered. Thus, a stimulus from an unexpected stimulus source should appear shorter.

However, there is some indirect evidence that the attenuation hypothesis does not apply to this situation when attention has to be shared between different stimulus sources. More specifically, the studies of Stelmach and co-workers (Stelmach \& Herdman, 1991; Stelmach, Herdman, \& McNeil, 1994) suggest the opposite prediction of the attenuation hypothesis, namely that stimuli at the expected location appear shorter than those at an unexpected location. Stelmach et al.'s hypothesis, which we will refer to as the temporal profile hypothesis, emerged from research on simultaneity judgments. Subjects had to judge whether two adjacent visual stimuli were simultaneous, and if not, which one was the first. Stimulus onsets were asynchronous with onset differences ranging from -100 to $100 \mathrm{msec}$. When attention was directed to one location by a cue, the overall percentage of simultaneous judgments decreased drastically. To account for this finding, they proposed a temporal profile model. According to this model, each stimulus elicits its own internal temporal response function (see, e.g., Ikeda, 1986; Roufs \& Blommaert, 1981). The model predicts that as the overlap of the two functions increases, the stimuli are more likely to be perceived as simultaneous. The authors attributed the decrease of simultaneous judgments under the directed-attention condition to a response function of the attended stimulus that is more brisk and reaches its peak sooner and, therefore, to a diminishing degree of overlap. The temporal profile model received further support from a recent study in which subjects were required to direct their overall level of attention toward both stimuli in a temporal order judgment task (Carver \& Brown, 1997).

The temporal profile model of Stelmach and colleagues (Stelmach \& Herdman, 1991; Stelmach et al., 1994) for the perception of simultaneity incorporates explicitly the notion that attention to a stimulus source shortens the duration of the perceptual event associated with the stimulus appearing at this location. Thus, the authors concluded that "for the attended stimulus processing reaches a peak sooner and the perceptual event has a shorter duration" (Stelmach et al., 1994, p. 108). Although the temporal profile model was proposed in the context of temporal order judgments, it nevertheless implies the hypothesis that directed attention should shorten perceived duration.

In conclusion, then, the attenuation hypothesis of Thomas and Weaver (1975) and the temporal profile hypothesis of Stelmach and colleagues (Stelmach \& Herdman, 1991; Stelmach et al., 1994) provide contradictory predictions on how directed spatial attention affects perceived duration. According to the attenuation hypothesis, attention acts to increase perceived duration, whereas the temporal profile hypothesis suggests that attention decreases perceived duration.
Relevant data for deciding between the two hypotheses were provided by Shore, Brehaut, and Enns (1997) and by Klein, Wylie, and Briand (1996). However, the findings of these studies are inconclusive. Shore et al. reported data consistent with the attenuation hypothesis, whereas the data of Klein et al. support neither the attenuation hypothesis nor the temporal profile hypothesis. The fact that Klein et al. failed to detect any effect of attention on perceived duration might, however, be due to methodological details in their task (Klein, 1997, personal communication). For example, lines were used as stimuli and subjects had to judge the duration of the line. If an attentional effect is restricted to a small area only, attention might not have spread over the whole length of the stimulus, with the result that an effect of spatial attention on perceived duration could not be detected.

The experiments described in this article were especially designed to test the attenuation hypothesis versus the temporal profile hypothesis. More specifically, we sought to investigate whether perceived duration increases or decreases with directed attention when it has to be divided between two stimulus sources. In Experiment 1, a precue indicated whether a stimulus was more likely to occur in the visual or in the auditory modality. The degree of attentional focusing was manipulated via the validity of the precue, and the subjects were required to rate the duration of the stimulus. Experiment 2 employed longer stimuli ( 200 instead of $70 \mathrm{msec}$ ) to assess whether the results obtained in Experiment 1 could be attributed to changes of apparent intensity. Experiment 3 employed a two-alternative forcedchoice task to assess whether the effects obtained so far were influenced by the specific method employed in the previous experiments. Whereas Experiments 1-3 employed intermodal stimulus sources, Experiments 4-6 employed different stimulus locations within the visual field to assess whether the attentional effect found for modality cuing generalizes to spatial cuing. A visual precue directed attention to the left or right of fixation. Figure 1 gives an overview of the experiments in this study.

\section{EXPERIMENT 1}

Experiment 1 adopted a rating procedure that has been employed before in time perception research (Long \& Beaton, 1980; Thomas \& Weaver, 1975). In each trial, one of two stimulus durations was presented either to the visual or to the auditory modality. The two durations were short and difficult for subjects to distinguish. A precue preceding the stimulus directed attention either to the visual or to the auditory modality, and precue validity ranged from 0.5 to 0.9 . Subjects were asked to judge the duration of the stimulus on a 3-point scale (short, medium, or long). It was assumed that subjects directed more attention to the precued sensory modality when precue validity increased. Accordingly, the manipulation of precue validity enabled us to assess whether directed attention shortens or lengthens the perceived duration of a stimulus. 
Experiments $1 \& 2$

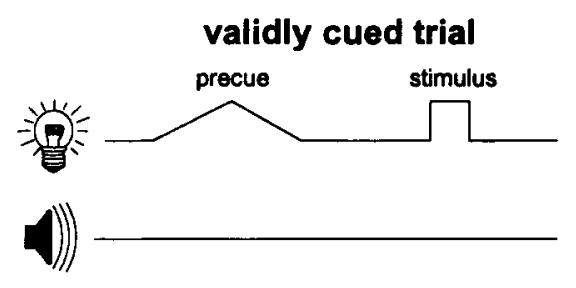

invalidly cued trial

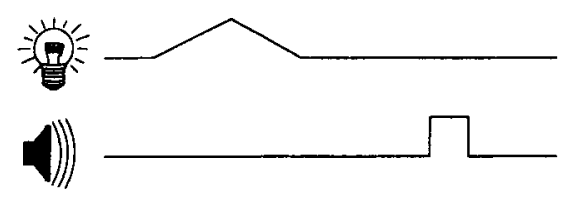

\section{Experiment 3}

validly cued trial

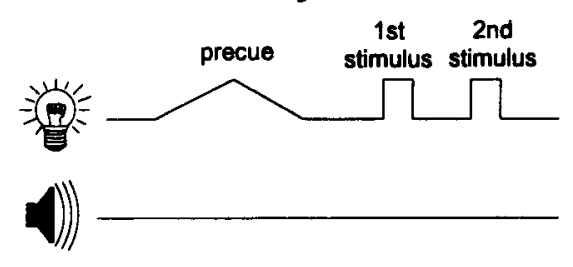

invalidly cued trial

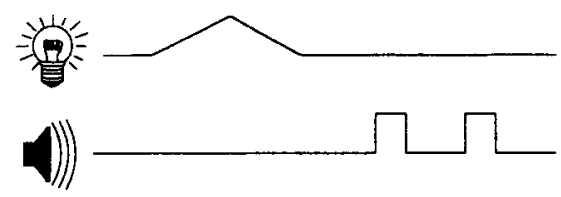

\section{Experiment 4}

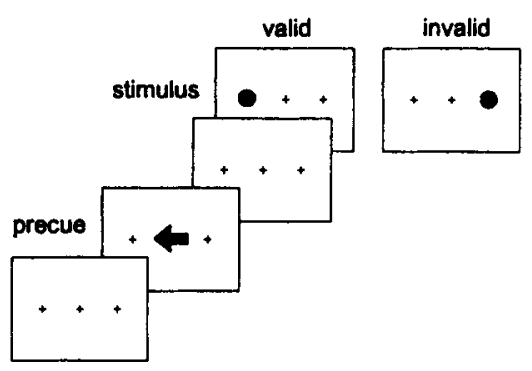

\section{Experiment 5}

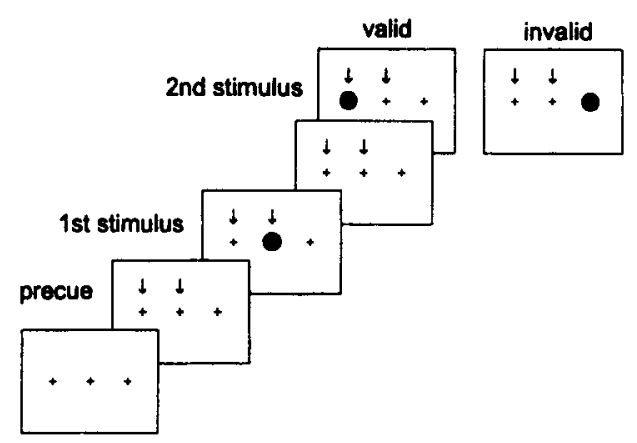

\section{Experiment 6}

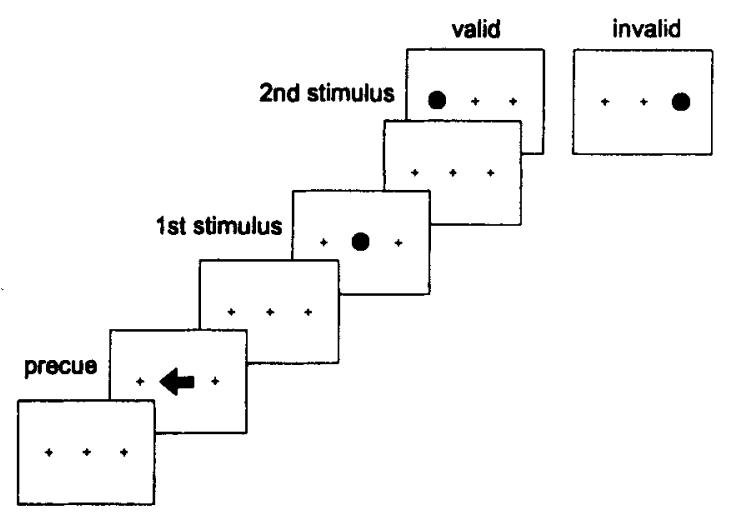

Figure 1. Illustration of the sequence of events for Experiments 1-6. Experiments 1-3 employed visual or auditory stimuli and attention was directed to either modality by a precue. Examples of validly and invalidly cued trials are depicted for each experiment. Experiments 4-6 employed visual stimuli and attention was directed to spatial locations by means of arrow precues. In valid trials, the stimulus or second stimulus was presented at the precued location, whereas in invalid trials, the stimulus appeared at the uncued location. Experiments 1, 2, and 4 employed a rating task in which a single stimulus had to be rated as short, medium, or long. Experiments 3, 5, and 6 employed a pair comparison task. Subjects had to judge whether the second of two stimuli was shorter or longer than the first.

\section{Method}

Subjects. Twelve subjects, 8 male and 4 female university students, were employed $1 \mathrm{~h}$ a day on 2 consecutive days. Their mean age was 26.3 years, with a range of $20-33$ years. They were paid for their cooperation and were naive about the purpose of the experiment.

Apparatus and Stimuli. A microcomputer controlled signal presentation and recorded ratings. A green LED (diameter $0.48^{\circ}$, viewing distance $60 \mathrm{~cm}$, luminance approximately $48 \mathrm{~cd} / \mathrm{m}^{2}$ ) marked the visual stimulus interval. The intensity of the LED was clearly above threshold, but not dazzling. The auditory stimulus consisted of a $1000-\mathrm{Hz}$ sinusoid tone of $75 \mathrm{~dB}$ (A) SPL and was presented via headphones. The duration of these stimuli was determined for each subject as described below. The LED and the headphones were also used to present precue information for the visual or the auditory modality. The precue signal for either modality rose and fell slowly over a total length of $1 \mathrm{sec}$ to provide a minimum of temporal information and was thus clearly distinguishable from the stimulus interval.

Procedure. A perceived-duration task (Thomas \& Weaver, 1975) was employed in which subjects had to rate the duration of 


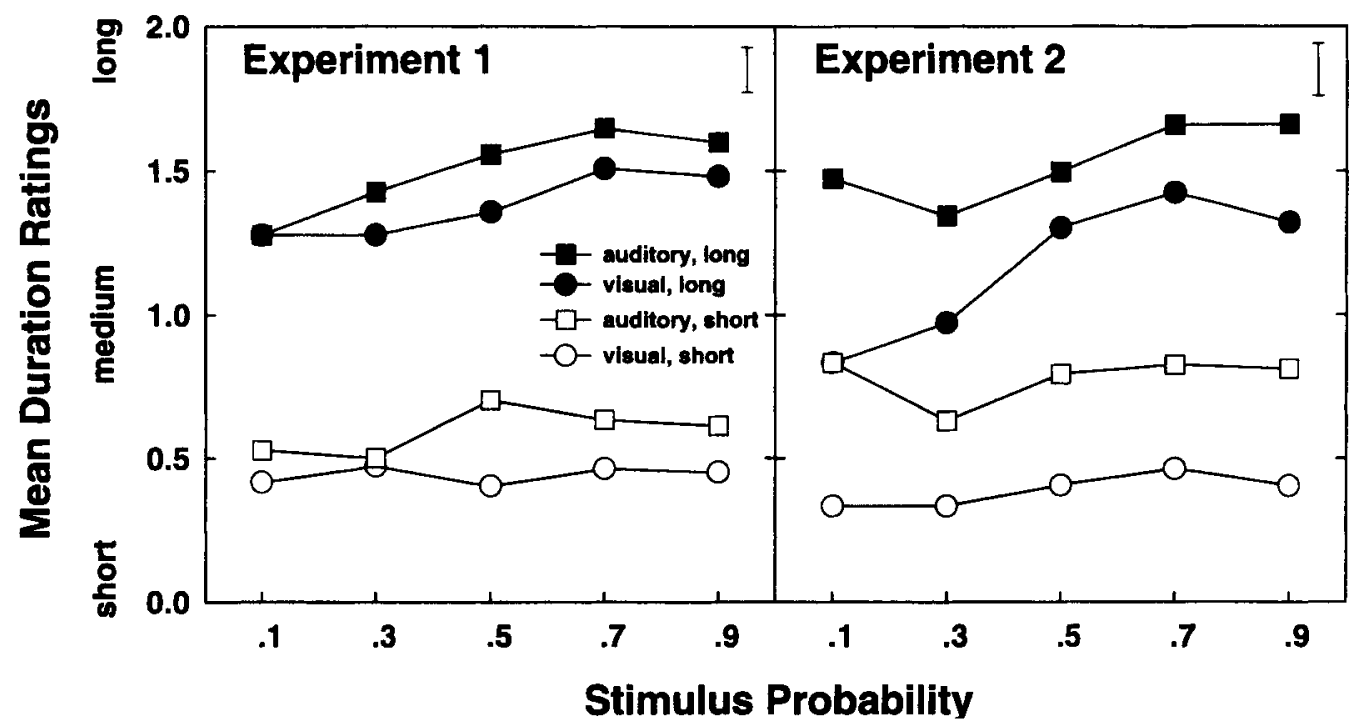

Figure 2. Mean duration ratings as a function of stimulus probability, sensory modality, and stimulus duration. The left panel shows the results of Experiment 1 and the right panel shows the results of Experiment 2. The error bar in the upper right corner of each panel indicates the standard error, which was estimated from the pooled error terms of the relevant ANOVA (Loftus \& Masson, 1994).

an auditory or a visual interval. Each trial started with the presentation of the visual or the auditory precue (Figure 1), each precue being equally likely. One second after precue offset, the stimulus to be judged was presented. The stimulus was either short or long and it was presented either in the visual or in the auditory modality. Subjects were asked to categorize the duration of the stimulus as short, medium, or long by pressing one of three keys with their index, middle, or ring finger of the right hand. Two seconds after the keypress, the next trial began.

The validity of the precue was kept constant within a single block and was $.9, .7$, or .5 . For example, a precue with a validity of .9 indicated that the stimulus would occur in the specified modality with a stimulus probability of .9 and in the nonspecified modality with the complementary probability of . 1 . The validity of the precue was presented visually on the computer screen before each block. The order of blocks with different precue validity was balanced over subjects. At the beginning of the experiment, subjects received a detailed explanation of the meaning of precue validity and were asked to direct their attention to the more likely stimulus.

The first session was subdivided into two parts. First, the difference limen (DL) was estimated for each modality and each subject so that the stimulus durations could be adjusted for the practice and experimental sessions according to the subject's performance; ${ }^{1}$ the duration of the short stimulus was always set to $70 \mathrm{msec}$ and the long duration to $(70+2 \cdot \mathrm{DL})$ msec. For the experimental session, the longer duration was readjusted if discrimination turned out to be too easy or too difficult. ${ }^{2}$ Averaged over all subjects, the long stimulus interval was $123 \mathrm{msec}(S D=29.8)$ for the visual modality and $80 \mathrm{msec}(S D=2.9)$ for the auditory modality, replicating the wellknown phenomenon that auditory intervals are easier to discriminate than visual ones (Goodfellow, 1934). In the second part of the first session, the subject was familiarized with the stimulus durations and received 20 presentations each of visual and auditory intervals, for which no rating was required. This presentation was followed by a further demonstration of 20 trials with a perfectly valid precue. The experimenter explained the meaning of the precue validities and checked whether the subject understood the task. Three practice blocks with 80 trials each followed; in each block a different precue validity was used.

The experimental session was administered on the following day. It consisted of three blocks with 160 trials each. The first 40 trials in each block were considered warm-up trials and thus not subjected to data analysis. Thus, in a block, there were 2 (stimulus modality: visual vs. auditory) $\times 2$ (stimulus duration: short vs. long) $\times 30=$ 120 regular trials, where the 30 consisted, depending on block, of (1) 3 invalidly cued and 27 validly cued (.9 proportion), (2) 9 invalidly cued and 21 validly cued (.7 proportion), or (3) 15 invalidly cued and 15 validly cued (.5 proportion).

Design. From the three precue validities $(.5, .7$, and .9), the following stimulus proportions emerged: $.1, .3, .5, .7$, and .9 . Thus, the experiment combined factorially the three within-subjects factors stimulus modality (visual vs. auditory), stimulus duration (short vs. long), and stimulus probability $(.1, .3, .5, .7$, vs. .9). The dependent variable was the mean rating of stimulus duration.

\section{Results}

The ratings "short," "medium," and "long" were coded as 0,1 , and 2, respectively. The left panel of Figure 2 shows the mean ratings as a function of stimulus probability, stimulus modality, and stimulus duration. A threeway analysis of variance (ANOVA) for these factors was performed on the ratings.

Mean ratings increased with stimulus probability, as revealed by a highly significant main effect $[F(4,44)=6.1$, $p<.01]$, indicating that perceived duration increased with stimulus probability; mean ratings were $0.88,0.92$, $1.01,1.06$, and 1.04 for the stimulus probabilities $.1, .3$, $.5, .7$, and .9 , respectively. The highly significant main effect of stimulus duration showed that discrimination of long versus short intervals was clearly above chance level $[F(1,11)=408.1, p<.001]$; mean ratings were 0.52 for 
short and 1.44 for long intervals. Comparison of the mean ratings for each modality yielded a tendency toward higher ratings for auditory intervals (1.05) than for visual intervals $(0.91)[F(1,11)=3.4, p<.10]$. The putative interaction of stimulus probability and stimulus duration indicated in Figure 2 was not significant $[F(4,44)=1.64$, $p<.20]$. However, separate ANOVAs for short and long intervals revealed no main effect of stimulus probability for the short interval $(F<1)$, but only for the long one $[F(4,44)=5.8, p<.01]$.

\section{Discussion}

The results support the hypothesis that perceived duration increases with directed attention because stimuli presented to the expected modality were judged longer than those presented to the unexpected modality. Moreover, this attentional effect was obtained for both visual and auditory stimuli.

\section{EXPERIMENT 2}

Most of the stimulus intervals employed in Experiment 1 were shorter than $100 \mathrm{msec}$. It is reasonable to assume that the perceived duration for such brief stimuli is influenced by apparent intensity differences (Bloch's law) or merely by differences in sensory persistence (Long \& Beaton, 1980). Though the relation of stimulus intensity and perceived duration is far from being clear (see Nisly \& Wasserman, 1989), it might be that subjects based their duration ratings on perceived intensity instead of perceived duration, or it might even be possible that the precue affected only sensory persistence. To rule out such alternative explanations, Experiment 2 employed longer stimulus intervals.

The procedure of Experiment 2 was identical to that of Experiment 1 except for stimulus duration. The durations used in this experiment were $200 \mathrm{msec}$ for the short interval and $(200+2 \cdot \mathrm{DL}) \mathrm{msec}$ for the long stimulus interval.

\section{Method}

Subjects. Twelve subjects ( 7 male, 5 female) were recruited for two sessions on different days. Their mean age was 25.1 years, with a range of 20-32 years. They were naive about the purpose of the experiment and none of them had participated in Experiment 1.

Stimuli, Procedure, and Design. The experiment was identical to Experiment 1 except for longer stimulus intervals. The short interval was 200 instead of $70 \mathrm{msec}$. The longer interval was again defined as $(200+2 \cdot D L)$ msec. The overall mean of the longer interval was $266 \mathrm{msec}(S D=22.8)$ for the visual modality and $229 \mathrm{msec}$ $(S D=10.3)$ for the auditory modality. As in Experiment 1, the smaller difference between short and long stimuli for the auditory modality showed that auditory stimuli were easier to discriminate than visual ones.

\section{Results}

Mean duration ratings are depicted in the right panel of Figure 2. As in Experiment 1, duration ratings increased with stimulus probability $[F(4,44)=11.0, p<.001]$; mean ratings were $0.87,0.82,1.00,1.09$, and 1.04 for the $.1, .3, .5, .7$, and .9 stimulus probabilities, respectively.
The main effect of stimulus duration was again significant $[F(1,11)=352.6, p<.001]$; mean ratings were 0.58 for short and 1.35 for long intervals. The significant interaction of stimulus probability and stimulus duration showed that the effect of stimulus probability on duration ratings was more pronounced for the long than for the short stimulus duration $[F(4,44)=3.5, p<.05]$. This interpretation of the interaction was confirmed by separate ANOVAs for short and long intervals. As in Experiment 1, stimulus probability significantly affected the ratings for the long interval $[F(4,44)=15.2, p<.001]$, but not for the short one $[F(4,44)=1.2, p>.3]$. Also in accord with Experiment 1, higher ratings were observed for auditory (1.15) than for visual (0.79) intervals. However, in contrast to Experiment 1, this difference attained statistical significance $[F(1,11)=17.2, p<.01]$.

\section{Discussion}

Experiment 2 replicated the main finding of Experiment 1 that duration ratings increase with stimulus probability. Hence, this finding is not restricted to intervals below $100 \mathrm{msec}$ and therefore rules out the alternative explanation, according to which subjects base their ratings on apparent stimulus intensity rather than on stimulus duration.

Auditory intervals were judged to have a longer duration than visual intervals, which might be attributed to longer auditory persistence. Such a difference has also been observed in other studies (e.g., Goldstone \& Goldfarb, 1964), though there seems to be conflicting data (Allan, 1979, p. 346).

So far, the results of Experiments 1 and 2 go against the temporal profile hypothesis, derived from the work of Stelmach and colleagues (Stelmach \& Herdman, 1991; Stelmach et al., 1994), according to which directing attention to a stimulus decreases its perceived duration.

\section{EXPERIMENT 3}

The preceding two experiments employed the same psychophysical method. Therefore, one might ask whether the results obtained so far are stable and not affected by methodological variation, because it is well known that results in time perception are subject to methodological influences (Allan, 1979; Zakay, 1993). Thus, Experiment 3 employed the method of pair comparison to assess whether the results obtained so far depend on the specific method employed or are unaffected by procedural change, as one would expect if the effect has a genuine perceptual basis.

The following paradigm was employed. Two visual or two auditory stimuli were presented successively (see Figure 1). The duration of the first stimulus was variable (comparison stimulus), whereas the second stimulus was a standard stimulus of constant duration. Subjects judged whether the second stimulus appeared to be longer or shorter than the first one. Analogous to Experiments 1 and 2 , a precue preceded the first stimulus and directed attention to one of the two possible stimulus modalities. It 
was assumed that the precue influences attention for the first but not the second stimulus; the second stimulus is always perceived with full attention since it is reliably presented in the same modality as the first stimulus. The precue had a validity of .7; that is, the stimulus probabilities were .7 for the cued and .3 for the noncued modality. In some trials, both precues were presented simultaneously, indicating that a stimulus will appear with a probability of .5 in either modality (neutral condition). Moreover, two standard durations (100 and $300 \mathrm{msec}$ ) were employed. Crossing of the factors standard duration and stimulus probability resulted in six conditions.

A psychometric function was generated for each condition and for each subject. For each psychometric function, the point of subjective equality (PSE) was estimated and employed to assess how attentional manipulation might influence perceived duration. We also estimated the DL to reveal whether the manipulations affected temporal discriminability.

\section{Method}

Subjects. A fresh sample of 10 subjects ( 5 male, 5 female) was tested. Each participated in four sessions on consecutive days. Their mean age was 24.1 years, with a range of $20-29$ years. All subjects were naive about the purpose of the experiment.

Apparatus and Stimuli. The visual and auditory stimuli as well as the precues were the same as those in Experiments 1 and 2. The standard durations were 100 and $300 \mathrm{msec}$. The comparison durations could be shorter or longer than the standards and were varied according to an adaptive rule (Kaernbach, 1991). For duration judgments, two keys on the computer keyboard were marked as "longer" and "shorter." The assignment of the keys was balanced over subjects.

Procedure. A trial started with the presentation of a visual or an auditory precue. In neutral trials, both precues were presented simultaneously. After an empty period of $1,500 \mathrm{msec}$, the comparison interval was presented either in the visual or in the auditory modality. Then, $1,500 \mathrm{msec}$ after the offset of the comparison interval, the standard interval was presented in the same modality as the comparison. The subjects were informed that the stimuli would appear in the precued modality with a probability of .7 and in the noncued with a probability of .3 . They were told to direct their attention always to the cued modality while keeping their eyes fixed on the LED. They were further informed that when both precues appeared, either modality was equally likely.

Subjects were required to indicate whether the second stimulus appeared shorter or longer than the first one. They responded by pressing the corresponding key ("shorter" vs. "longer") on the keyboard (two-alternative forced-choice technique). The next precue was presented $500 \mathrm{msec}$ after the keypress.

Each subject served in four sessions; the first one was considered practice. One session was performed per day and consisted of 480 trials. The standard duration changed after 240 trials, and this change was announced to the subject. One third of the trials was assigned to the neutral condition, in which both precues were presented simultaneously. Half of the subjects started with the short standard in the first session. The order of the standard duration changed from one session to the next session. The duration of the comparison stimulus was changed according to an adaptive rule (Kaernbach, 1991) to estimate $x_{.25}$ and $x_{.75}$, that is, the two comparison intervals at which the response "longer" was given with the probability .25 or .75 . To estimate $x_{.25}$, the duration of the comparison interval was increased by $5 \mathrm{msec}$ if the subject had judged the standard to be longer and decreased by $15 \mathrm{msec}$ after a short judg- ment. The opposite step sizes were employed for $x_{75}$. Initial durations of the comparison were $30 \mathrm{msec}$ below and above the standard stimulus for $x_{-25}$ and $x_{7_{75}}$. This procedure was performed for each of the six conditions ( 3 stimulus probabilities $\times 2$ stimulus modalities), resulting in 12 randomly interleaved trial runs. Thus, the factor stimulus duration was blocked, whereas the factors stimulus modality and stimulus probability were randomly mixed.

Estimation of PSE, constant error (CE), and DL. A maximum likelihood procedure was used to determine PSE $=x_{.50}$ and $\mathrm{DL}=\left(x_{\cdot 75}-x_{\cdot 25}\right) / 2$ for each level of stimulus probability and standard duration. To this end, a logistic psychometric function (see Bush, 1963),

$$
\text { Prob("longer"| } \left.x_{i}\right)=\frac{1}{1+e^{\left(\operatorname{PSE}-x_{i}\right) /(0.91 \cdot \mathrm{DL})}},
$$

was employed, which associates the comparison interval $x_{i}$ in the $i$ th trial with the probability of the response "longer." The estimates of PSE and DL were those values that maximized the corresponding likelihood function. Pilot studies and simulations indicated that this estimation procedure produces relatively low variance estimates of both DL and PSE. To simplify matters, the CE (PSE - POE) was used for further analysis as a measure of the deviation of the PSE from the point of objective equality ( $\mathrm{POE}$ ), which corresponds to the duration of the standard interval. A positive (negative) CE indicates that the comparison interval had to be increased (decreased) relative to the standard interval in order for both intervals to be perceived as equally long.

Design. Three within-subjects factors were factorially combined: stimulus probability $(.3, .5$, and .7$)$, stimulus duration (short vs. long), and stimulus modality (visual vs. auditory). Dependent variables were mean CE and mean DL for each subject and each factorial combination.

\section{Results}

The first 40 trials for each standard duration were considered warm-up trials and excluded from data analysis. Separate three-way ANOVAs with the factors stimulus probability, standard duration, and stimulus modality were performed for CE and DL.

CE. The upper panel of Figure 3 shows the mean CE as a function of stimulus probability, standard duration, and stimulus modality. There was a highly significant main effect of stimulus probability on $\mathrm{CE}[F(1,18)=6.8$, $p<.01]$; as expected, the mean CE decreased with stimulus probability $(4.65,0.83$, and -4.23$)$. Consistent with the attentional effect of the previous experiments, the comparison was perceived to be longer when it occurred in the expected modality. CE did not differ significantly for visual and auditory stimuli ( -0.24 vs. $1.07 ; F<1)$. For the short standard duration, CE was negative $(-4.49)$, whereas for the long standard duration it was positive (5.43). This means that the first interval was generally judged as longer than the second interval for durations of about $100 \mathrm{msec}$, whereas the opposite pattern was observed for durations of about $300 \mathrm{msec}$. This reversal is in accordance with findings on the so-called time-order error (Block, 1994); however, this main effect failed to reach significance $[F(1,9)=3.8, p<.1]$.

There was a significant interaction of stimulus probability and stimulus duration $[F(2,18)=4.8, p<.05]$. Separate ANOVAs for the short and the long standard intervals confirmed what the figure suggests: There was a 


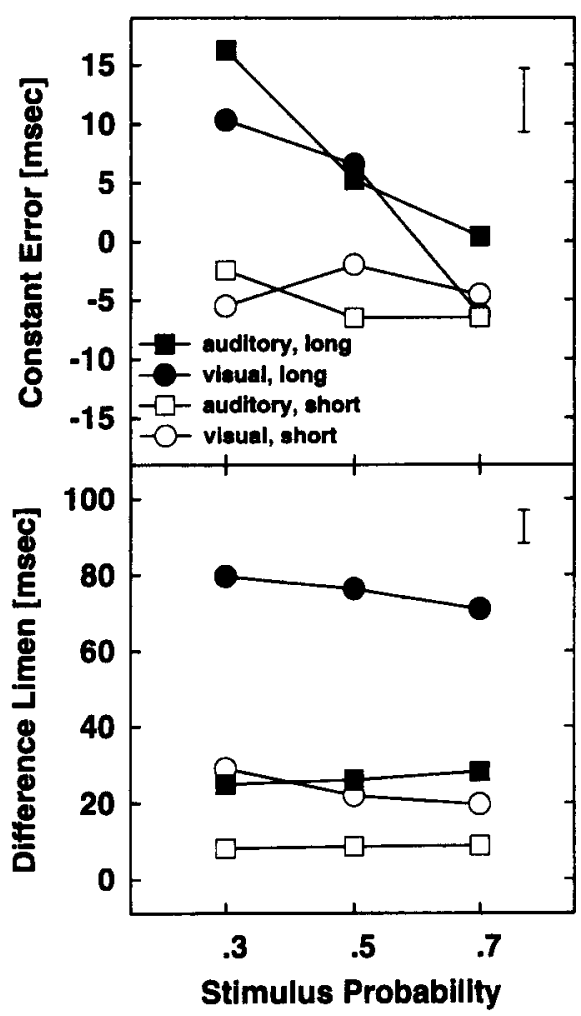

Figure 3. Constant error (upper panel) and difference limen (lower panel) for Experiment 3 as a function of stimulus probability, standard duration, and stimulus modality. High values for constant error indicate a subjective shortening of the interval. The error bar in the upper right corner of each panel indicates the standard error.

clear main effect of stimulus probability for the longer standard, with mean CEs of 13.3, 5.9, and $-2.9[F(2,18)=$ $6.5, p<.01]$, whereas there was virtually no effect for the short standard interval $(-4.0,-4.2,-5.5, F<1)$.

DL. The lower panel of Figure 3 shows mean DL as a function of stimulus probability, standard duration, and stimulus modality. Although DL does not assess perceptual bias, it nevertheless seems interesting to see how the manipulations affected temporal discriminability. Therefore, an ANOVA analogous to that used for the CE was carried out for DL. As in the preceding experiments, subjects more accurately discriminated among auditory stimuli than among visual ones [ 17.4 vs. $49.6 \mathrm{msec}, F(1,9)=$ $18.9, p<.01]$. Shorter stimuli were more accurately discriminated than longer ones $[F(1,9)=18.3, p<.01]$. However, as indicated by a significant modality $\times$ duration interaction $[F(1,9)=7.1, p<.05]$, this duration effect on DL was more pronounced for visual than for auditory stimuli. This interaction can be attributed to Weber's law (DL/standard = constant), which predicts a larger absolute increase in DL with standard duration when the level of DL is already high than when it is low. Indeed, the Weber fractions for auditory stimuli were almost identical for both standard durations $(8.5 / 100=0.09 ; 26.4 / 300=0.09)$, and an analogous constancy applies to visual stimuli $(23.5 / 100=0.24 ; 75.6 / 300=0.25)$. Temporal discrimination did not depend on precue information $(F<1)$. No other effects reached significance.

\section{Discussion}

The results of Experiment 3 confirm the findings of both previous experiments. Again, perceived duration of a stimulus increased when attention was directed to the source of the upcoming stimulus, at least when longer stimuli were employed. This replication of the attentional effect on perceived duration makes it less likely that this effect was due to a methodological artifact. As in the previous experiments, the precue information affected the duration judgments for long but not for short durations, suggesting that attention does not influence perceived duration for very brief stimuli.

\section{Intermediate Summary}

In Experiments 1-3, subjects were instructed to direct attention to one of two sensory modalities and judge the duration of a stimulus appearing in the attended or unattended modality. Although the three experiments were different with regard to the psychophysical method employed, the common finding is that judged duration increases when attention is directed to the modality of a stimulus. This result is consistent with the prediction of the attenuation hypothesis but at variance with the temporal profile hypothesis.

The next three experiments assessed whether the same pattern of results would emerge when the precue indicates different locations within the visual field. Experiment 4 employed the rating procedure of Experiments 1 and 2, and Experiments 5 and 6 used the pair comparison method employed in Experiment 3.

\section{EXPERIMENT 4}

The procedure of this experiment was identical to that of Experiments 1 and 2 except that the factor stimulus modality (visual vs. auditory) was replaced by the factor stimulus location (left vs. right). An arrow served as a precue and pointed to the more likely stimulus location to the right or the left of a fixation point.

\section{Method}

Subjects. A fresh sample of 7 male and 5 female subjects was tested in two sessions on consecutive days. Their mean age was 24.3 years, with a range of $20-32$ years. They were naive about the purpose of the experiment.

Apparatus and Stimuli. A microcomputer controlled signal presentation and recorded ratings. The stimuli were presented on an NEC Multisync 4FG monitor with a viewing distance of $60 \mathrm{~cm}$. The stimuli were black dots with a diameter of $1.4^{\circ}$ and a luminance of $0.1 \mathrm{~cd} / \mathrm{m}^{2}$ on a gray background with a luminance of $11 \mathrm{~cd} / \mathrm{m}^{2}$ (see Figure 1). There was a fixation cross in the middle of the screen. One stimulus location was $3.6^{\circ}$ to the left and the second one $3.6^{\circ}$ to the right of the central fixation point. Each stimulus location was marked by a small cross. The precue was an arrow (height $0.4^{\circ}$, width $0.76^{\circ}$ ) presented at the fixation point and point- 


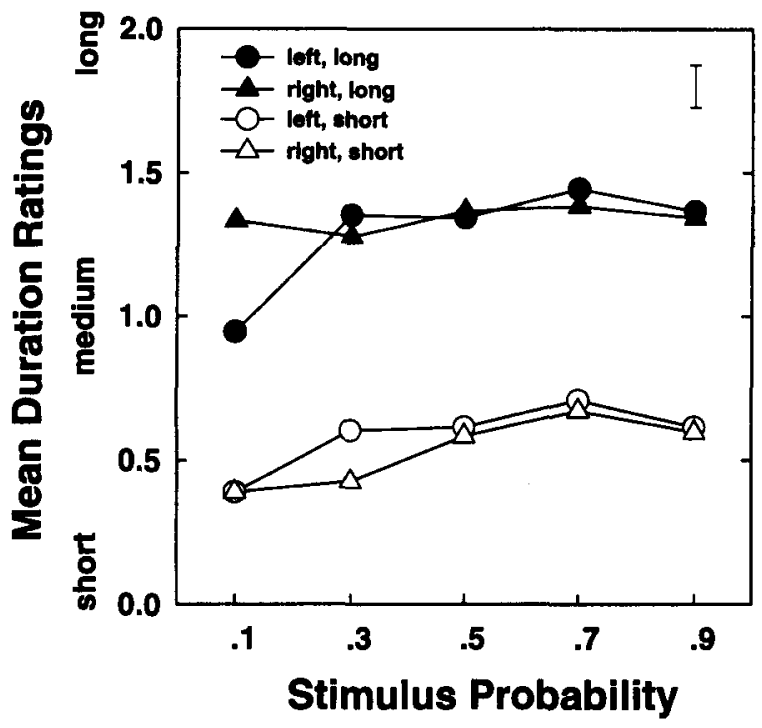

Figure 4. Mean duration ratings as a function of stimulus probability, stimulus duration, and side for Experiment 4. The error bar in the upper right corner indicates the standard error.

ing either to the left or to the right. The location markers and precues had the same luminance as the stimuli. Owing to the monitor's refresh rate of $70 \mathrm{~Hz}$, stimulus duration could be varied in steps of about $14 \mathrm{msec}$ only. The short stimulus was set to $200 \mathrm{msec}$.

Procedure and Design. The procedure and the design were virtually identical to those of Experiments 1 and 2 except that factor stimulus modality (visual vs. auditory) was now replaced by stimulus location (left vs. right). As in the previous experiments, the precue rose and fell over a duration of $1 \mathrm{sec}$ to provide a minimum of temporal information. One second later the short or the long stimulus appeared at the left or the right stimulus location. Subjects were advised to fix their eyes on the central cross during the trial.

As in Experiments 1 and 2, the DL was determined for each subject before the practice and experimental sessions. For all subjects, the short interval was $200 \mathrm{msec}$, and the longer interval was tailored according to the performance of each subject to be $(200+2$. DL) msec. The resulting overall mean duration of the longer stimulus was thus $231 \mathrm{msec}(S D=11.2)$ for the experimental session.

\section{Results}

Figure 4 depicts mean duration ratings as a function of stimulus probability and stimulus duration. There was again a highly significant main effect of stimulus probability on mean duration ratings $[F(4,44)=6.95, p<.01]$. Consistent with Experiments 1 and 2, mean duration rating increased with stimulus probability $(0.76,0.91,0.98$, 1.05 , and 0.98$)$. A trend analysis revealed both a significant linear trend $[F(1,44)=19.1, p<.001]$, and a significant quadratic trend $[F(1,44)=7.9, p<.01]$. The quadratic trend is apparent in a somewhat convex shape of the probability function (see Figure 4). Mean ratings for the short and long intervals were 0.56 and 1.32 , respectively $[F(1,11)=111.8, p<.001]$. Ratings for stimuli presented to the left and to the right were almost identical $(0.938$ vs. $0.937, F<1)$. However, there was a significant interaction of stimulus side and stimulus duration $[F(1,11)=$
$5.4, p<.05]$; stimuli presented to the right side were easier to discriminate $(0.53$ vs. 1.34 , for the short and long stimuli, respectively) than stimuli presented to the left side $(0.59$ vs. 1.29$)$. This nicely agrees with the notion that the left cerebral hemisphere is superior to the right in time discrimination (for a review, see Nicholls, 1996).

\section{Discussion}

The present experiment confirmed the main finding of the previous ones. As before, stimuli appearing at the precued stimulus location were judged to be longer than stimuli appearing at the uncued location. Therefore, the attentional effect on perceived duration found for the intermodal task in Experiments 1 to 3 seems to have generalized to an intramodal task.

\section{EXPERIMENT 5}

This experiment sought to further verify the effect of spatial attention with a pair comparison task, similar to the procedure employed in Experiment 3, in which a stimulus was presented in either the visual or the auditory modality. Two visual stimuli appeared on the screen and subjects had to judge whether the second stimulus appeared to be shorter or longer than the first one (see Figure 1). The standard stimulus was always presented at a central fixation point, whereas the comparison stimulus appeared either to the left or to the right of the fixation point.

A precue preceded the standard stimulus and directed attention to one of the two possible stimulus locations. The precue, an arrow above one of the two possible locations of the comparison stimulus, had a validity of .7; that is, the stimulus probabilities were .7 for the cued and .3 for the noncued location. Again, two standard durations ( 100 and $300 \mathrm{msec}$ ) were employed. Crossing of the factors standard duration and stimulus probability resulted in four conditions.

Subjects were also required to respond as quickly as possible to the onset of the comparison with a keypress (simple reaction time $[\mathrm{RT}]$ ) before indicating their duration judgment. Simple RT has been shown to be sensitive to attentional precuing of this kind (Posner, Nissen, \& Ogden, 1978). Thus, RT should provide independent evidence that the precues directed attention to the desired location.

\section{Method}

Subjects. Ten subjects ( 5 male, 5 female) were tested. Each participated in four sessions on consecutive days. Their mean age was 26.8 years, with a range of $21-31$ years. All subjects were naive about the purpose of the experiment.

Apparatus and Stimuli. The stimuli were similar to those employed in Experiment 4. The stimulus locations were marked with three small crosses (about $0.3^{\circ}$ ), one at the center of the screen and the others to either side at a distance of $3.6^{\circ}$ from the center. Precues were arrows above the possible stimulus locations with a length of $2.2^{\circ}$. Their heads pointed to the marker crosses at a distance of $2^{\circ}$. Markers and precues had the same luminance as stimuli. The standard durations were 100 and $300 \mathrm{msec}$. The comparison durations 


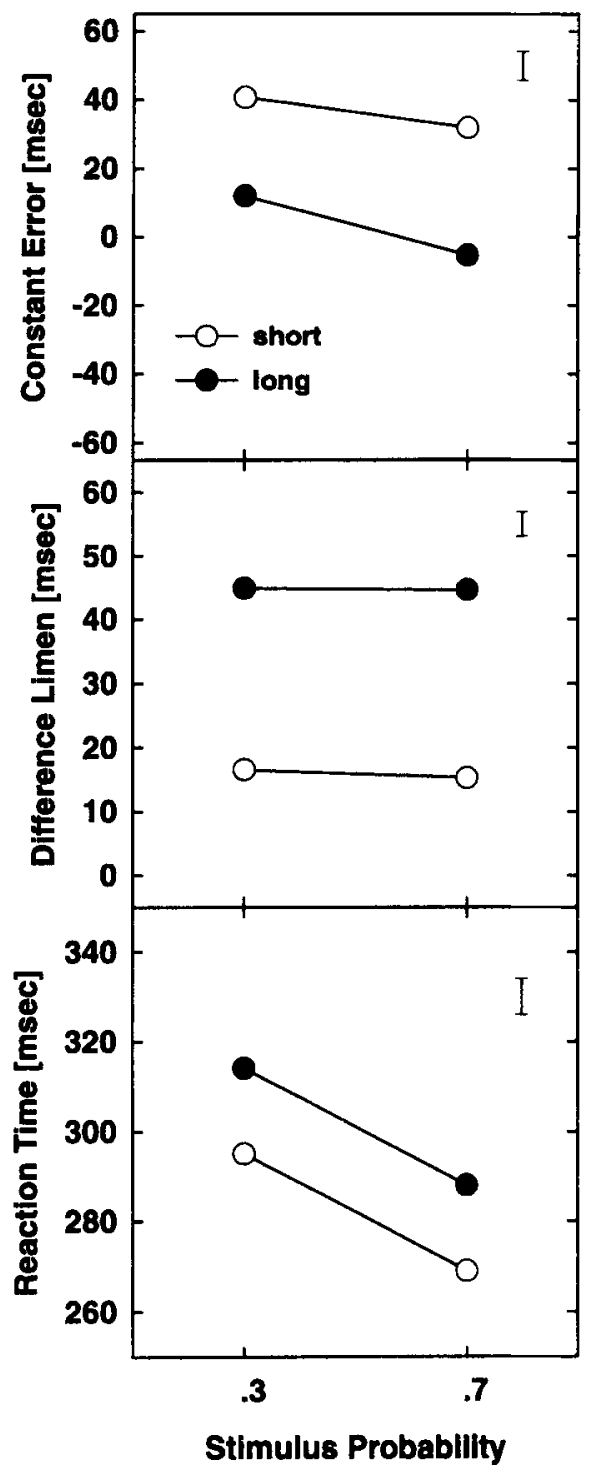

Figure 5. Constant error (upper panel), difference limen (middle panel), and reaction time (lower panel) for Experiment 5 as a function of stimulus probability and standard duration. High values for constant error indicate a subjective shortening of the interval. The error bar in the upper right corner of each panel indicates the standard error.

were varied according to the same adaptive rule as that in Experiment 3. For duration judgments, two keys on the computer keyboard were marked as "longer" and "shorter." The assignment of the keys was balanced over subjects. Simple RTs were measured with a response key that looked like a telegraph key

Procedure. A short beep indicated the beginning of a trial and told the subjects to fix their eyes on the central marker. Then, $300 \mathrm{msec}$ after the beep, two arrows were presented above two of the three crosses, one of them always above the center cross, while the other arrow, the precue, specified the more likely stimulus location of the comparison stimulus. After a period of $1,500 \mathrm{msec}$, the standard interval was presented at the center, superimposed on the cross. Then, $1,500 \mathrm{msec}$ after the offset of the standard interval, the comparison interval was presented at the left or the right marker.
The subjects were informed that the comparison appeared at the precued location with a probability of .7 and at the noncued one with a probability of .3 . Subjects were told to direct their attention always to the cued location while keeping their eyes fixed on the center. The subjects were asked for a simple reaction when they detected the comparison stimulus. After this simple RT task, subjects were required to provide their duration judgment ("shorter" or "longer") by pressing the corresponding key on the keyboard. The next trial started when the subject released the key.

The number of sessions and number of trials per session were identical to those of Experiment 3. To estimate $x_{25}$, according to the adaptive rule the duration of the comparison interval was now increased by $14 \mathrm{msec}$ when the subject judged the comparison stimulus as shorter than the standard and decreased by $42 \mathrm{msec}$ when the comparison stimulus was judged longer. The reverse figures were applied to estimate $x_{.75}$. Initial durations of the comparison stimulus were $42 \mathrm{msec}$ above and below the standard stimulus for $x_{.75}$ and $x_{.25}$, respectively. PSE, CE, and DL were estimated the same way as in Experiment 3.

Design. Three within-subjects factors were factorially combined: stimulus probability ( .3 vs. . 7), stimulus duration (short vs. long), and stimulus location (left vs. right). Dependent variables were mean $\mathrm{CE}$, mean DL, and mean RT for each subject and each factorial combination.

\section{Results}

CE. The first 40 trials for each standard duration were considered warm-up trials and were excluded from data analysis. Separate three-way ANOVAs with the factors stimulus probability, standard duration, and stimulus location were performed for CE, DL, and RT. The upper panel of Figure 5 shows the mean $\mathrm{CE}$ as a function of stimulus probability and standard duration. The overall mean CE was $19.8 \mathrm{msec}$, reflecting the usual underestimation of the second of two short intervals (Allan, 1977). As in other studies (e.g., Rammsayer, 1992), this tendency diminished as the duration of the standard increased $[F(1,9)=12.8, p<.01]$. Most important, however, there was a highly significant main effect of stimulus probability on $C E[F(1,9)=15.6, p<.01]$; as expected, the mean CE was smaller for the more likely stimulus location $(\mathrm{CE}=13.3$ vs. $26.4 \mathrm{msec})$; consistent with the attentional effect of the previous experiments, the comparison stimulus was perceived to be longer when it occurred at the cued stimulus location. Although Figure 5 suggests that the attentional effect on CE was less pronounced for short stimuli than for longer stimuli, this effect was not statistically reliable $[F(1,9)=1.8, p>.2]$. No other effect was significant.

DL. As in Experiment 3, an ANOVA was performed for DL to reveal the effects on temporal discriminability of the factors employed (Figure 5). As expected, DL increased with standard duration $[F(1,9)=88.5, p<.001]$, with larger DLs for the longer than for the shorter standard interval ( $44.8 \mathrm{vs} .15 .8 \mathrm{msec})$. These estimates are in agreement with Weber's law $(44.8 / 300=0.15 ; 15.8 / 100=$ $0.16)$. As in Experiment 3, stimulus probability did not influence temporal discriminability $(F<1)$.

RT. As can be seen in Figure 5, stimulus probability showed the expected effect on RT, with faster RTs for stimuli with higher probability $[278$ vs. $304 \mathrm{msec}, F(1,9)=$ $47.3, p<.001]$. This agrees with RT studies on visual at- 
tention (e.g., Posner et al., 1978) and provides strong evidence that the subjects directed their attention to the cued stimulus location.

\section{Discussion}

The results of Experiment 5 clearly confirm and further strengthen the conclusion of the previous experiments that perceived duration of a stimulus increases when attention is directed to the upcoming stimulus. The present replication of the attentional effect on perceived duration makes it less likely that the effect is due to a methodological artifact. Furthermore, the relatively short RTs for cued stimuli show that the subjects followed the instructions and directed their attention to the cued stimulus location.

\section{EXPERIMENT 6}

The main purpose of the following experiment was to ensure that the results in the previous experiments with intramodal stimulation were not subject to an eye movement artifact. One might argue that some eye movements toward the precued stimulus location occurred in some of the trials, even though subjects were advised to fix their eyes on the central point. In this case, the validly cued stimuli would stimulate the fovea, whereas the invalidly cued stimuli would stimulate more peripheral areas of the retina. Thus, the precue effect obtained in the intramodal experiments might rather reflect temporal differences of retinal eccentricities (see Hollmann, 1985). Therefore, in this experiment eye movements were recorded and trials showing eye movements were discarded from the data analysis. If the effects in the preceding two experiments were due to eye movements, the precue effect on judged duration should disappear when only trials without eye movements are included in the data analysis.

\section{Method}

Subjects. A fresh sample of 2 male and 4 female subjects was tested in three sessions on consecutive days. Their mean age was 25.8 years, with a range of $21-31$ years. All subjects were naive about the purpose of the experiment.

Apparatus and Stimuli. The same stimuli as those in Experiment 5 were used except for the precue, which was now a central arrow, as in Experiment 4 (see Figure 1).

Recording of eye movements. Horizontal electroocculograms (EOGs) were recorded bipolar from the outer canthi with one-way $\mathrm{Ag} / \mathrm{AgCl}$ electrodes (ARBO, Type H207P). Recording started at precue onset and ended $1 \mathrm{sec}$ after the offset of the comparison stimulus. The sampling rate was $500 \mathrm{~Hz}$ and the signal was filtered (.01$\mathrm{Hz}$ high-pass, $100-\mathrm{Hz}$ low-pass) and stored for further analysis.

Procedure. The procedure was similar to that of Experiment 5 except for four changes. First, no simple RT task was employed. Second, only one standard duration $(200 \mathrm{msec})$ was used. Third, a central precue, as in Experiment 4, was used to deter subjects from making eye movements. Finally, each subject was tested in three sessions on consecutive days. Subjects were explicitly instructed not to move their eyes during the interval between precue onset and offset of the comparison.

Design. Stimulus probability (.3 vs. .7) and stimulus location (left vs. right) were factorially combined. The dependent variables were again $C E$ and DL.

\section{Results}

Each trial was visually inspected for horizontal eye movements. Trials with eye movements were excluded from further analysis. The percentage of trials with eye movements was $0.6 \%$. Separate two-way ANOVAs with the factors stimulus probability and stimulus location were performed for CE and DL. It turned out that stimuli at precued locations were again judged as longer than uncued stimuli even when the influence of eye movements was excluded. The respective CEs were $3.1 \mathrm{msec}$ for stimuli appearing at the likely location and $6.7 \mathrm{msec}$ for stimuli at the unlikely location $[F(1,5)=7.2, p<.05]$. Neither the main effect of factor stimulus location nor the interaction of the two factors was significant. The DLs for high and low stimulus probabilities were virtually identical ( 14.9 vs. $14.4 \mathrm{msec}, F<1$ ).

\section{Discussion}

The outcome of this experiment shows that the effect of stimulus probability on judged duration is not due to an eye movement artifact. The effect was somewhat smaller than that in Experiment 5. Although it is generally difficult to compare effect sizes across experiments, subjects might have paid less attention to the precue because no speeded responses were required, and this might have diminished the attentional effect on perceived duration. Nevertheless, the effect obtained replicated the basic finding of all the previous experiments, namely that stimuli from attended sources are perceived as longer than stimuli from unattended sources.

\section{Summary}

In Experiments 4-6, subjects had to direct attention in advance to one of two possible locations within the visual field. Although the three experiments differed in several aspects (psychophysical method, arrangement of precues, inclusion of simple RT task), the same main conclusion emerges: Judged duration increases when attention is directed to the location of a stimulus.

\section{GENERAL DISCUSSION}

In this paper, we have sought to assess whether or not directed attention to a precued stimulus source affects the perceived duration of a stimulus appearing within this source. More specifically, we were interested in finding out whether the perceived duration of a stimulus appearing in the more attended stimulus source increases or decreases. As discussed in the introduction, the attenuation hypothesis (Thomas \& Weaver, 1975) holds that perceived duration increases with the amount of attention devoted to the expected stimulus. In contrast, the temporal profile hypothesis (Stelmach \& Herdman, 1991; Stelmach et al., 1994) suggests that a stimulus in the attended source appears shorter than one appearing in the unattended source.

In each experiment of this study, manipulation of stimulus expectation clearly affected judged duration. Subjects generally judged a stimulus as longer when it appeared 
at the precued stimulus source than when it appeared at the uncued one. Taken as a whole, this main result clearly supports the attenuation hypothesis but is at variance with the temporal profile hypothesis.

This main result proved to be fairly stable. First, it was obtained when subjects divided attention between the auditory and visual modality (Experiments 1-3) and between two spatial locations within the visual field (Experiments $4-6$ ). Second, the effect was obtained with different psychophysical procedures. It was established with a rating procedure (Experiments 1,2, and 4), but also with a pair comparison task (Experiments 3, 5, and 6). However, stimulus duration seems to have influenced the cuing effect on judged duration. In particular, when attention was directed to the visual or auditory modality in the bimodal attention task, cuing had a greater effect on long than on short stimuli (Experiments 1-3, but not Experiments 4 and 5). This interaction is, however, consistent with the finding that the perception of brief durations is less or even not at all sensitive to experimental manipulations than the perception of longer durations (Rammsayer, 1996). Furthermore, similar interactions have been reported in studies that used the same method (Long \& Beaton, 1980; Thomas \& Cantor, 1975) but that assessed the effect of stimulus size instead of stimulus probability on perceived duration. Thus, it also seems possible that this interactive effect might reflect a peculiarity of this psychophysical method. It must be stressed, however, that this interaction is not particularly relevant in discriminating between the two competing hypotheses.

One might doubt that the present precue effect reflects a perceptual effect and argue that it is rather a responsebias effect. Accordingly, it might be generally assumed that, for whatever reason, subjects tend to choose the response "shorter" for the less frequent stimulus. Such a bias could explain the finding that stimuli at uncued sources are judged as shorter than stimuli at cued sources without involving perceptual processes. However, there is evidence that argues against such an account. In a recent study, Tse, Intriligator, Cavanagh, and Rivest (1997) presented a stream of standard stimuli of equal duration. A stimulus differing in motion, color, or size was randomly inserted into this stream. Contradicting the account given above, subjects judged the odd and thus less frequent item to last longer than standard stimuli of the same duration.

A related account proposes that subjects generally tend to give the response "longer" for validly precued trials. However, this bias account can be refuted by the outcome of Experiment 3 . If subjects generally tend to give the response "shorter" in invalidly cued trials, then the opposite result should have emerged in this experiment, namely that cued stimuli are judged to be shorter. In this experiment, the first of two intervals was precued and subjects had to judge whether the second interval appeared shorter or longer than the first one. If in validly cued trials subjects pressed the "longer" key more often, this would imply that the first interval is judged shorter than invalidly cued trials. However, this was not observed, which provides strong evidence against this account. Therefore, the present main result that validly cued stimuli were judged longer than invalidly cued stimuli is difficult to explain with a response-bias account, strengthening the argument that the present main effect reflects a genuine perceptual effect.

The present results do not support the notion suggested by Stelmach and co-workers (Stelmach \& Herdman, 1991; Stelmach et al., 1994) that attention shortens the internal representation of an attended stimulus. These authors argued on the basis of results obtained from temporal-order judgments that a stimulus is processed faster when attention is directed to it. As discussed in the introduction, subjects judged whether two visual stimuli were simultaneous or, if not, which one was the first. When attention was directed to one stimulus, subjects gave fewer simultaneous but more successive judgments. To explain this shift, they proposed that the internal temporal profile of a stimulus decreases as more attention is devoted to this stimulus and that the degree of perceived simultaneity increases with the overlap of the temporal response functions of two stimuli. Because the temporal response function of the attended stimulus decreases, the degree of overlap diminishes.

It is, however, possible to reconcile the basic idea of the temporal profile model by assuming that attention does not decrease the internal representation of a stimulus but rather increases it. Accordingly, the response function of an attended stimulus would increase relatively steeply and reach a higher maximum level. If the decay rate were the same for both attended and unattended stimuli, the profile function of the attended stimulus would be longer than that of the unattended stimulus. Therefore, the overlap would decrease as more attention is directed to one stimulus, and this would also account for Stelmach et al.'s simultaneity results. Furthermore, this modified version of their model would account for their temporal-order judgment results because the difference function of the two temporal response functions has virtually the same properties as in the original model (see Stelmach \& Herdman, 1991). Thus, the modified model would be consistent with both the results of Stelmach et al. and the present results that attention prolongs the perceived duration of a stimulus. Further research is clearly needed to assess this modification in more detail.

The attentional effect revealed in the present experiments agrees with that found in the dual-task studies mentioned in the introduction, namely that an attended stimulus is perceived to be longer than an unattended one. But contrary to those earlier studies, the present paradigm did not require sharing of attention between a temporal and a nontemporal task. Instead, a precue directed attention to one of two possible stimulus sources. Hence, it also seemed interesting to see whether the general finding of dual-task studies would generalize to a situation in which attention has to be directed in advance to one of two possible stimulus sources and not to one of two competing tasks. 
Although the present findings indicate that such a generalization is valid, the explanation of the present attentional effects within the framework of the attenuation hypothesis is somewhat difficult. As noted, the attenuation hypothesis attributes the attentional effect on perceived duration to changes within the internal clock mechanism. In traditional dual-task studies, it is assumed that some clicks are lost when attention has to be shared with a nontemporal task. Therefore, an interval is underestimated when more attention has to be devoted to the nontemporal task. However, it seems difficult to attribute the attentional effect obtained in this study to the diminished efficiency of an internal clock mechanism because no nontemporal task was involved that could detract attention capacity from the internal clock. One might, however, argue that a stimulus appearing in an unattended location requires an especially large amount of central capacity to be processed and, therefore, some clicks get lost.

In conclusion, then, the present study reveals how the amount of attention directed to a particular input source influences the perceived duration of a stimulus appearing within this source. The attenuation hypothesis leads us to expect that perceived duration will increase when more attention is directed to a particular source, whereas the temporal profile hypothesis suggests that perceived duration will decrease. The whole pattern of the present results clearly shows that judged duration increases with the amount of attention devoted to the relevant input source. Therefore, the present results provide evidence against the temporal profile hypothesis but support the attenuation hypothesis. Nevertheless, it is possible to reconcile the temporal profile model with the present results, as we have argued.

There is an important theoretical difference between the attenuation hypothesis and the modified profile model. The attenuation hypothesis suggests that the attentional effect on perceived duration is direct because attention directly affects the efficacy of the internal clock. In contrast, however, the modified temporal profile model suggests an indirect effect of attention on perceived duration since attention should not affect the internal clock per se but rather the duration of the internal stimulus representation on which the internal clock operates. More research is needed to distinguish between such direct and indirect effects.

\section{REFERENCES}

AllaN, L. G. (1977). The time-order error in judgments of duration. Canadian Journal of Psychology, 31, 24-31.

Allan, L. G. (1979). The perception of time. Perception \& Psychophysics, 26, 340-354.

Block, R. A. (1994). Time-order errors. In S. L. Macey (Ed.), Encyclopedia of time (pp. 632). New York: Garland.

Brown, S. W., \& WEST, A. N. (1990). Multiple timing and the allocation of attention. Acta Psychologica, 75, 103-121.

BuSH, R. R. (1963). Estimation and evaluation. In R. D. Luce, R. R. Bush, \& E. Galanter (Eds.), Handbook of mathematical psychology (Vol. 1, pp. 429-469). New York: Wiley.

CARVER, R. A., \& Brown, V. (1997). Effects of amount of attention al- located to the location of visual stimulus pairs on perception of simultaneity. Perception \& Psychophysics, 59, 534-542.

Creelman, C. D. (1962). Human discrimination of auditory stimuli. Journal of the Acoustical Society, 34, 582-593.

Goldstone, S., \& GoldFarb, J. L. (1964). Direct comparison of auditory and visual durations. Journal of Experimental Psychology, 67, 483-485.

GOODFELLOW, L. D. (1934). An empirical comparison of audition, vision, and touch in the discrimination of short intervals of time. American Journal of Psychology, 46, 243-258.

Grondin, S., \& MaCar, F. (1992). Dividing attention between temporal and nontemporal tasks: A performance operating characteristicPOC-analysis. In F. Macar, V. Pouthas, \& W. Friedman (Eds.), Time, action and cognition: Towards bridging the gap (pp. 119-128). Dordrecht: Kluwer.

HollmanN, A. (1985). Zeitwahrnehmung in Abhängigkeit von der retinalen Exzentrizität [The relation between time perception and retinal eccentricity]. Unpublished doctoral dissertation, Ludwig-MaximiliansUniversität, Munich.

HÜLSER, C. (1924). Zeitauffassung und Zeitschätzung verschieden ausgefüllter Intervalle unter besonderer Berücksichtigung der Aufmerksamkeitsablenkung [Time perception and time estimation of filled intervals when attention is distracted]. Archiv der gesamten Psychologie, 49, 363-378.

IKEDA, M. (1986). Temporal impulse response. Vision Research, 26, $1431-1440$.

JAMES, W. (1890). The principles of psychology. New York: Holt.

KaERNBACH, C. (1991). Simple adaptive testing with the weighted up-down method. Perception \& Psychophysics, 49, 227-229.

Kahneman, D. (1973). Attention and effort. Englewood Cliffs, NJ: Prentice-Hall.

Katz, D. (1906). Experimentelle Beiträge zur Psychologie des Vergleichs im Gebiete des Zeitsinns [Experimental contributions to the psychology of our sense of time]. Zeitschrift für Psychologie, Physiologie der Sinnesorgane, 42, 302-340.

Klein, R. M., Wylie, G., \& Briand, K. (1996, October). How does covert visual orienting affect absolute judgments of size and duration? Paper presented at the annual meeting of the Psychonomic Society, Chicago.

Loftus, G. R., \& MAsson, M. E. J. (1994). Using confidence intervals in within-subjects designs. Psychonomic Bulletin \& Review, 1, 476-490.

LONG, G. M., \& BEATON, R. J. (1980). The contribution of visual persistence to the perceived duration of brief targets. Perception \& Psychophysics, 28, 422-430.

Macar, F., Grondin, S., \& CASINI, L. (1994). Controlled attention sharing influences time estimation. Memory \& Cognition, 22, 673-686.

NiCHOLLS, M. E. R. (1996). Temporal processing asymmetries between the cerebral hemispheres: Evidence and implications. Laterality, 1, 97-137.

NisLy, S. J., \& WASSERMAN, G. S. (1989). Intensity dependence of perceived duration: Data, theories, and neural integration. Psychological Bulletin, 106, 483-496.

Posner, M. I., Nissen, M. J., \& Ogden, W. C. (1978). Attended and unattended processing modes: The role of set for spatial location. In H. L. Pick, Jr., \& I. J. Saltzman (Eds.), Modes of perceiving and processing information (pp. 137-157). Hillsdale, NJ: Erlbaum.

PREDEBon, J. (1996). The relationship between the number of presented stimuli and prospective duration estimates: The effect of concurrent task activity. Psychonomic Bulletin \& Review, 3, 376-379.

QUASEBARTH, K. (1924). Zeitschätzung und Zeitauffassung optisch und akustisch ausgefüllter Intervalle [Time estimation and time perception of optically and acoustically filled intervals]. Archiv der gesamten Psychologie, 49, 379-432.

RAMMSAYER, T. (1992). Die Wahrnehmung kurzer Zeitdauern [The perception of short temporal intervals]. Münster: Waxmann.

Rammsayer, T. (1996). Experimental evidence for different timing mechanisms underlying temporal discrimination. In S. Masin (Ed.), Fechner Day 96: Twelft h annual meeting of the International Society for Psychophysics (pp. 63-68). Padua: International Society for Psychophysics. 
Roufs, J. A. J., \& Blommaert, F. J. J. (1981). Temporal impulse and step responses of the human eye obtained psychophysically by means of a drift-correcting perturbation technique. Vision Research, 21, 1203-1221.

ShORE, D. I., Brehaut, J., \& ENNS, J. T. (1997). The duration of a brief event in the mind's eye. Investigative Ophthalmology \& Visual Science, 38, 1798.

Stelmach, L. B., \& Herdman, C. M. (1991). Directed attention and perception of temporal order. Journal of Experimental Psychology: Human Perception \& Performance, 17, 539-550.

Stelmach, L. B., Herdman, C. M., \& McNeil, K. R. (1994). Attentional modulation of visual processes in motion perception. Journal of Experimental Psychology: Human Perception \& Performance, 20, 108-121.

Thomas, E. A. C., \& Cantor, N. E. (1975). On the duality of simultaneous time and size perception. Perception \& Psychophysics, 18, 44-49.

Thomas, E. A. C., \& CaNToR, N. E. (1978). Interdependence between the processing of temporal and non-temporal information. In J. Requin (Ed.), Attention and performance VII (pp. 43-62). Hillsdale, NJ: Erlbaum.

Thomas, E. A. C., \& Weaver, W. B. (1975). Cognitive processing and time perception. Perception \& Psychophysics, 17, 363-367.

Treisman, M. (1963). Temporal discrimination and the indifference interval: Implications for a model of the "internal clock." Psychological Monographs, 77, 1-31.

Tse, P., InTriligator, J., Cavanagh, P., \& Rivest, J. (1997). Attention distorts the perception of time. Investigative Ophthalmology \& Visual Science, 38, 5379.
UNDERWoOD, G., \& SWAIN, R. A. (1973). Selectivity of attention and the perception of duration. Perception, 2, 101-105.

ZAKAY, D. (1993). Time estimation methods: Do they influence prospective duration estimates? Perception, 22, 91-101.

ZAKAY, D., \& TsAL, Y. (1989). Awareness of attention allocation and time estimation accuracy. Bulletin of the Psychonomic Society, 27, 209-210.

\section{NOTES}

1. The DL estimation was based on an interval discrimination task with a fixed standard $(70 \mathrm{msec})$ and a variable comparison interval. Because an adaptive technique was employed, reliable estimations were obtained in 72 trials per modality. The adaptive method was similar to the one described in the method section of Experiment 3.

2. For the experimental (second) session, the duration of the longer interval was adjusted according to the following rule: $d_{2}=d_{1} * 0.7 /\left(L_{1}-\right.$ $S_{1}$ ), where $d_{2}$ and $d_{1}$ are the stimulus durations of the long interval in the first and second sessions, and $L_{1}$ and $S_{1}$ are the mean judgments for the long and short intervals in the first session. In other words, if the difference in judgments for short and long intervals in the first session was less than 0.7 , the duration of the longer interval was increased to increase discriminability, whereas if the difference was more than 0.7 , the longer interval was made shorter to decrease discriminability. The same adjustment was employed in Experiments 2 and 4.

(Manuscript received March 11, 1997; revision accepted for publication October 24, 1997.) 